\title{
FRACIONAMENTO DE METAIS PESADOS EM SOLO CONTAMINADO ANTES E APÓS CULTIVO DE ARROZ
}

\author{
Maria Ligia de Souza Silva* e Godofredo Cesar Vitti \\ Departamento de Ciência do Solo, Escola Superior de Agricultura "Luiz de Queiroz", Universidade de São Paulo, CP 9, \\ 13418-900 Piracicaba - SP, Brasil \\ Recebido em 12/7/07; aceito em 15/2/08; publicado na web em 31/7/08
}

\begin{abstract}
FRACTIONATION OF HEAVY METALS IN POLLUTED SOIL BEFORE AND AFTER RICE CULTIVATION. The objective of the present work was to evaluate the distribution of $\mathrm{Cd}, \mathrm{Cu}, \mathrm{Mn}, \mathrm{Pb}$ and $\mathrm{Zn}$ among the different fractions of contaminated soil, before and after rice cultivation. Seven soil samples with different degrees of contamination were studied using a randomized experimental design, with four replicates. Using an ICP-OES we analyzed the contents of heavy metals in fractions of soil, the organic matter therein, the oxides and the residual content before and after rice cultivation. The largest concentrations of $\mathrm{Cd}, \mathrm{Cu}, \mathrm{Mn}$ and $\mathrm{Zn}$ in the soil are found in the fractions with stable chemical bonds.
\end{abstract}

Keywords: chemical extractors; pollution; sequential extraction.

\section{INTRODUÇÃO}

A crescente industrialização tem levado à contaminação de solos por diferentes metais pesados, sendo que esses solos passam a prejudicar o desenvolvimento normal de muitas espécies de plantas, bem como a própria microbiota do solo. ${ }^{1}$ Alem da contaminação do solo, cursos de água e lençol freático também podem ser atingidos pela contaminação, provocando sérios problemas ambientais. ${ }^{2}$

Os metais pesados, quando aplicados aos solos, podem acumular e persistir por longos períodos de tempo e podem, além de fitotóxicos, serem prejudiciais a processos microbianos vitais na ciclagem de nutrientes. ${ }^{3}$

A toxicidade e a mobilidade dos metais pesados dependem fortemente da sua forma química e das ligações especificas (precipitados com minerais primários ou secundários, complexados por ligantes orgânicos, entre outros). Mudanças nas condições ambientais, tais como acidificação, mudanças dos potenciais de oxiredução ou aumento da concentração dos ligantes orgânicos podem causar a mobilização dos metais pesados da fase sólida para a fase líquida, favorecendo assim uma possível contaminação às plantas cultivadas nesses solos. ${ }^{4}$

Uma das ferramentas para se avaliar o comportamento dos metais pesados no solo é a utilização dos chamados métodos de extração seqüencial. Conceitualmente, os metais pesados contidos no material sólido podem ser fracionados em formas geoquímicas específicas e ser seletivamente extraídos pelo uso de reagentes apropriados. Cada método apresenta um conjunto de reagentes, que devem atacar especificamente a forma geoquímica para o qual está quimicamente ligado a estrutura do solo. ${ }^{5}$ As informações obtidas permitem avaliar a fitodisponibilidade, fitotoxicidade, dinâmica e as transformações dos metais pesados entre as diferentes formas químicas em solos contaminados, que um dia foram ou serão agricultáveis. ${ }^{6}$

O princípio da extração seqüencial seria que elementos cada vez mais imóveis podem ser extraídos por reativos cada vez mais eficientes. ${ }^{7}$ Alguns autores consideram a extração seqüencial como a determinação das formas químicas de metais pesados em solos e sedimentos, enquanto outros consideram que o método trata do fracionamento do solo através de reativos químicos ou solventes,

*e-mail:mlsousi@hotmail.com denominados extratores, para extrair elementos ligados ou associados a uma fase ou composto particular do solo. ${ }^{5,8}$

Um dos primeiros métodos desenvolvidos possui o esquema de fracionamento mais utilizado atualmente, onde utiliza diferentes soluções, dividindo os metais pesados nas seguintes frações: trocável, ligados a carbonato, ligados a óxidos de ferro e manganês, ligados à matéria orgânica e residual..$^{5}$ Outro esquema de fracionamento foi proposto para estudar metais pesados em solos ácidos, que não contêm carbonatos e quantidades apreciáveis de sulfeto. ${ }^{9}$ Neste esquema, são contempladas as frações: trocável, ligada à matéria orgânica e a óxidos de $\mathrm{Mn}$ e Fe (cristalinos e amorfos) e residual.

Em experimento com amostras de Latossolo Vermelho-Amarelo Húmico incubado com soluções de sais de cádmio, crômio, cobre, níquel, chumbo e zinco foi proposta uma metodologia para solos tropicais. ${ }^{10}$ Este esquema de fracionamento se tornou o mais adequado para solos de regiões tropicais, pois, entre outras vantagens, elimina a fração ligada a carbonato e inclui os óxidos de Al juntamente com os de Fe e Mn. ${ }^{10} \mathrm{Em}$ solos de regiões tropicais a fração ligada a carbonato não possui representatividade, em relação à fração ligada a óxidos de $\mathrm{Fe}$ e $\mathrm{Al}$, devido a esses solos possuírem alta intemperização.

Baseando-se em varias revisões, pesquisadores propuseram método de fracionamento com ordem de extração mais adequada e com mínimas chances do extrator retirar elementos de outra fração. ${ }^{11}$

Esse trabalho teve como objetivo avaliar a distribuição de Cd, $\mathrm{Cu}, \mathrm{Mn}, \mathrm{Pb}$ e $\mathrm{Zn}$ entre as diferentes frações de um solo contaminado por estes elementos, antes do cultivo (solo inicial) e após o cultivo de arroz

\section{PARTE EXPERIMENTAL}

O experimento foi conduzido em casa de vegetação do Departamento de Ciência do Solo da Escola Superior de Agricultura "Luiz de Queiroz", no período de novembro de 2004 a abril de 2005.

O solo, classificado como Latossolo Vermelho distroférrico de textura argilosa, ${ }^{12}$ utilizado no estudo foi coletado próximo a uma fábrica, no município de Paulínia, SP, a qual utilizava resíduos para fabricação de seus produtos e cuja contaminação ocorreu por via eólica. Foram coletados em 7 pontos distanciados em 100 m, partindo da 
fonte de poluição, na profundidade de $0-20 \mathrm{~cm}$. De cada ponto foram retiradas 3 amostras, as quais foram secas ao ar e passadas por peneira de $2 \mathrm{~mm}$ e analisadas quanto ao $\mathrm{pH}\left(\mathrm{CaCl}_{2}\right)$, matéria orgânica (MO), fósforo $(\mathrm{P})$, potássio $(\mathrm{K})$, cálcio $(\mathrm{Ca})$, magnésio $(\mathrm{Mg})$, acidez ativa $(\mathrm{H}+\mathrm{Al})^{13} \mathrm{e}$ teores totais de $\mathrm{Cd}, \mathrm{Cu}, \mathrm{Mn}, \mathrm{Pb}$ e $\mathrm{Zn}^{14}$ (Tabela 1).

$\mathrm{O}$ experimento foi conduzido em delineamento inteiramente casualizado, com 4 repetições, em vasos com capacidade para $5 \mathrm{dm}^{3}$. A espécie utilizada no experimento foi a Oriza sativa L. cultivar IAC 202. A adubação NPK foi realizada conforme necessidade da cultura, baseando-se nas recomendações do Boletim 100. ${ }^{15}$ Foram semeadas 10 sementes por vaso e após 15 dias da germinação realizou-se desbaste deixando 4 plantas por vaso. As plantas foram conduzidas até maturação. A irrigação dos vasos foi realizada de modo a não ultrapassar o limite máximo de $70 \%$ e mínimo de $40 \%$ da capacidade de retenção de água do solo, com pesagem diária dos vasos.

No procedimento de extração seqüencial aplicado para o fracionamento dos metais pesados presentes nas amostras de solo, antes do cultivo (solo inicial) e após o cultivo de arroz, foi excluída a fase carbonato, que é sabido ser muito pequena no solo estudado.

O fracionamento consistiu de quatro extrações em sequiência, sendo a primeira à fração solúvel + trocável, a segunda ligada à fração orgânica, a terceira ligada à fração oxídica e a quarta ao resíduo, segundo metodologia de Ahnstrom e Parker. ${ }^{11}$ As amostras foram moídas em almofariz de porcelana para promover melhor homogeneização de seus componentes e facilitar a ação das soluções extratoras.

$\mathrm{O}$ fracionamento foi conduzido utilizando-se amostras de $2 \mathrm{~g}$ de terra em tubos de centrifuga de $50 \mathrm{~mL}$, onde:

Fração 1 - metais pesados na fração solúvel + trocável: para a extração dos elementos da fração solúvel + trocável, adicionaramse $15 \mathrm{~mL}$ de $\operatorname{Sr}\left(\mathrm{NO}_{3}\right)_{2} 0,1 \mathrm{~mol} \mathrm{~L}^{-1}$ aos tubos, agitando por $2 \mathrm{~h} \mathrm{em}$ baixa velocidade. Após, centrifugou-se a $2500 \mathrm{rpm}$ durante $10 \mathrm{~min}$ e o sobrenadante, filtrado com papel de filtro quantitativo faixa azul, foi recolhido em balão volumétrico de $50 \mathrm{~mL}$. Repetiu-se o procedimento.

Fração 2 - metais pesados na fração orgânica: para a extração dos metais pesados da fração orgânica foram utilizados $5 \mathrm{~mL}$ de $\mathrm{NaOCl} 5 \%$, com pH ajustado para 8,5 com $\mathrm{HNO}_{3}$ concentrado. Os tubos foram colocados em banho-maria mantendo-se a temperatura de $95{ }^{\circ} \mathrm{C}$ durante 30 min sob agitação lenta. Após esse tempo, as amostras foram centrifugadas a $2500 \mathrm{rpm}$ durante 10 min e o sobrenadante, filtrado com papel de filtro quantitativo faixa azul, recolhido em balão volumétrico de $50 \mathrm{~mL}$. O procedimento foi repetido até a queima da matéria orgânica, que ocorreu quando cessou a formação de espuma.

Fração 3 - metais pesados na fração oxídica: para extração dos metais pesados ligados à fração oxídica foram adicionados $20 \mathrm{~mL}$ de uma mistura de 0,2 mol L $\mathrm{L}^{-1}$ de oxalato de amônio, 0,2 mol L $\mathrm{L}^{-1}$ de ácido oxálico e $0,1 \mathrm{~mol} \mathrm{~L}^{-1}$ de ácido ascórbico com $\mathrm{pH}$ ajustado para 3,0 com $\mathrm{NH}_{4} \mathrm{OH}$ concentrado. Os tubos foram colocados em banho-maria mantendo-se a temperatura de $95^{\circ} \mathrm{C}$ durante $30 \mathrm{~min}$ sob agitação lenta. Após esse tempo, as amostras foram centrifugadas a $2500 \mathrm{rpm}$ durante 10 min e o sobrenadante, filtrado com papel de filtro quantitativo faixa azul, recolhido em balão volumétrico de 100 $\mathrm{mL}$. As extrações foram realizadas até a cor do solo ficar cinza (sem ferro) e o sobrenadante amarelado.

Ao final de cada processo de extração, nas frações 1,2 e 3 , as amostras de solo foram lavadas com $5 \mathrm{~mL}$ de $\mathrm{NaCl} 0,1 \mathrm{~mol} \mathrm{~L}^{-1}$, centrifugadas a $2500 \mathrm{rpm}$ durante $10 \mathrm{~min}$, e os sobrenadantes recolhidos junto ao extrato referente à fração. A esse extrato adicionou-se $1 \mathrm{~mL}$ de $\mathrm{HNO}_{3}$ concentrado para a preservação, e completou-se o volume com água deionizada.

Fração 4 - metais pesados na fração residual: as amostras de solo foram colocadas para secar em estufa a $60{ }^{\circ} \mathrm{C}$ durante $24 \mathrm{~h} \mathrm{e}$ retiradas dos tubos de centrifuga. Fez-se a extração da fração residual com água régia (mistura de 3:1 de $\mathrm{HCl} / \mathrm{HNO}_{3}$ ), em sistema aberto, utilizando método modificado. ${ }^{16}$ Adicionaram-se $12 \mathrm{~mL}$ de $\mathrm{HCl}$ e 3 $\mathrm{mL}$ de $\mathrm{HNO}_{3}$, as amostras foram aquecidas por $3 \mathrm{~h}$ à temperatura de $60{ }^{\circ} \mathrm{C}, 1 \mathrm{~h}$ à $105^{\circ} \mathrm{C}$ e $30 \min$ à $140{ }^{\circ} \mathrm{C}$. Deixou-se esfriar e o sobrenadante, filtrado com papel de filtro quantitativo faixa azul, foi recolhido em balão volumétrico de $50 \mathrm{~mL}$. Completou-se o volume com água deionizada.

Os teores de $\mathrm{Cd}, \mathrm{Cu}, \mathrm{Mn}, \mathrm{Pb}$ e $\mathrm{Zn}$ nos extratos de cada fração foram determinados por espectrofotometria de emissão por plasma induzido de argônio (ICP-OES). As concentrações dos metais pesados foram analisadas estatisticamente e comparadas pelo teste de Tukey a $5 \%$ através do programa estatístico SAS. ${ }^{17}$

Tabela 1. Atributos químicos do solo e teores $\left(\mathrm{mg} \mathrm{kg}^{-1}\right)$ totais e disponíveis dos metais pesados no solo para cada tratamento

\begin{tabular}{|c|c|c|c|c|c|c|c|c|}
\hline \multicolumn{9}{|c|}{ Pontos de coleta de solo } \\
\hline Determinação & Unidade & 1 & 2 & 3 & 4 & 5 & 6 & 7 \\
\hline $\mathrm{pH} \mathrm{CaCl}_{2}$ & & 5,8 & 5,7 & 5,2 & 5,5 & 5,4 & 5,3 & 5,2 \\
\hline MO & $\mathrm{g} \mathrm{dm}^{-3}$ & 34,8 & 36,3 & 34,4 & 37,2 & 35,4 & 36,3 & 33,5 \\
\hline $\mathrm{P}$ & $\mathrm{g} \mathrm{dm}^{-3}$ & 59,1 & 36,0 & 56,1 & 22,1 & 21,5 & 20,7 & 17,5 \\
\hline K & $\mathrm{mmol}_{\mathrm{c}} \mathrm{dm}^{-3}$ & 12,0 & 14,1 & 11,4 & 11,8 & 11,3 & 12,4 & 11 \\
\hline $\mathrm{Ca}$ & $\mathrm{mmol}_{\mathrm{c}} \mathrm{dm}^{-3}$ & 41 & 39 & 38 & 40 & 36 & 36 & 37 \\
\hline $\mathrm{Mg}$ & $\mathrm{mmol}_{\mathrm{c}} \mathrm{dm}^{-3}$ & 18 & 19 & 20 & 22 & 20 & 19 & 21 \\
\hline $\mathrm{H}+\mathrm{Al}$ & $\mathrm{mmol}_{\mathrm{c}} \mathrm{dm}^{-3}$ & 28 & 33 & 32 & 29 & 29 & 31 & 31 \\
\hline SB & $\mathrm{mmol}_{\mathrm{c}} \mathrm{dm}^{-3}$ & 71,0 & 72,1 & 69,4 & 73,8 & 67,3 & 67,4 & 69,0 \\
\hline $\mathrm{T}$ & $\mathrm{mmol}_{\mathrm{c}} \mathrm{dm}^{-3}$ & 99,0 & 105,1 & 101,4 & 102,8 & 96,3 & 98,4 & 100,0 \\
\hline $\mathrm{V}$ & $\%$ & 71,7 & 68,6 & 68,4 & 71,8 & 69,9 & 68,5 & 69,0 \\
\hline $\mathrm{Cd}$ & $\mathrm{mg} \mathrm{kg}^{-1}$ & 24,7 & 23,3 & 20,4 & 23,3 & 26,3 & 27,9 & 26,7 \\
\hline $\mathrm{Cu}$ & $\mathrm{mg} \mathrm{kg}^{-1}$ & 272,1 & 140,8 & 115,0 & 120,6 & 144,1 & 166,1 & 153,3 \\
\hline $\mathrm{Fe}$ & $\mathrm{mg} \mathrm{kg}^{-1}$ & 332,9 & 208,0 & 173,5 & 198,4 & 226,1 & 244,1 & 228,6 \\
\hline Mn & $\mathrm{mg} \mathrm{kg}^{-1}$ & 539,6 & 562,7 & 535,6 & 582,7 & 664,4 & 719,2 & 570,5 \\
\hline $\mathrm{Pb}$ & $\mathrm{mg} \mathrm{kg}^{-1}$ & 332,9 & 208,0 & 173,5 & 198,4 & 226,1 & 244,1 & 228,6 \\
\hline $\mathrm{Zn}$ & $\mathrm{mg} \mathrm{kg}^{-1}$ & 553,6 & 188,7 & 112,8 & 105,7 & 105,6 & 108,0 & 102,0 \\
\hline
\end{tabular}




\section{RESULTADOS E DISCUSSÃO}

\section{Cádmio (Cd)}

A distribuição do Cd, nas diversas formas químicas, não apresentou variações significativas entre os pontos de coleta no solo inicial. No entanto, observa-se que após o cultivo de arroz houve variação entre os pontos de coleta de solo (Figura 1).
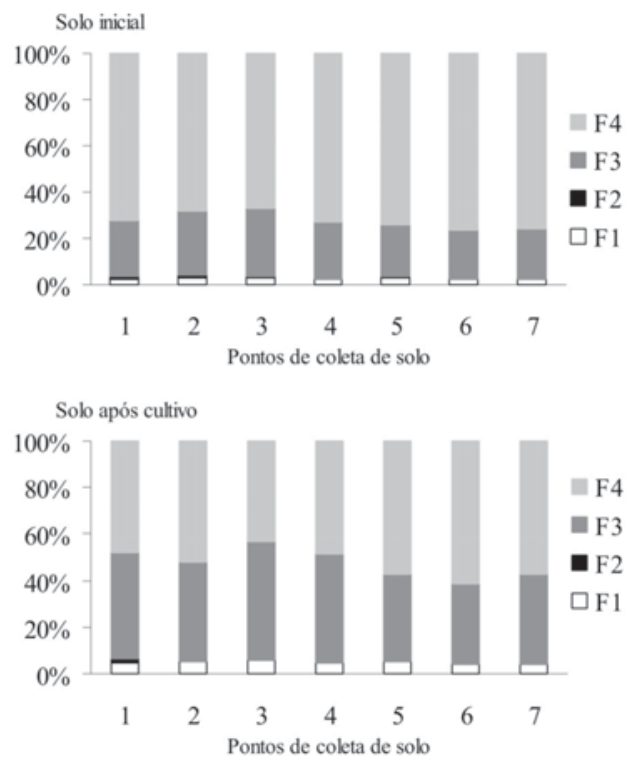

Figura 1. Distribuição do Cd, em porcentagem, nas frações do solo (fração solúvel - F1, fração ligada à matéria orgânica - F2, fração ligada a óxidos - F3 e fração residual - F4) nos pontos de coleta de solo

É evidente a menor distribuição de Cd nas frações solúvel + trocável e ligada à matéria orgânica, em todos os pontos de coleta no solo inicial. No solo após o cultivo de arroz observa-se ligeiro aumento da porcentagem de Cd nestas frações. Estas formas encontram-se ligadas por forças eletrostática nas cargas negativas da argila e da matéria orgânica do solo.

Quantidades consideráveis foram extraídas pelas frações ligadas a óxidos de $\mathrm{Fe}$ e $\mathrm{Al}$ e residual em todos os pontos de coleta, demonstrando a importante participação dos óxidos de Fe na adsorção desse elemento em solos com baixo teor de matéria orgânica. Após o cultivo do arroz observa-se que houve aumento na porcentagem de $\mathrm{Cd}$ ligada a óxidos de Fe do ponto 7 para o ponto 1, do mais distante para o mais próximo à fonte de poluição, sendo que o ponto 3 foi o que apresentou maior porcentagem. Nos solos sob clima tropical, os óxidos de $\mathrm{Fe}$ e $\mathrm{Al}$ exercem importante papel no comportamento dos metais pesados. A adsorção desses elementos aos óxidos de $\mathrm{Al}$ pode ocorrer por meio da formação de ligações covalentes com o $\mathrm{OH}^{-}$e/ou $\mathrm{O}^{2-}$ na superfície desses colóides. ${ }^{18} \mathrm{Em}$ menores valores de $\mathrm{pH}$ pode haver maior oxidação da matéria orgânica, liberando o Fe complexado. ${ }^{19}$ Esse fato poderia justificar o aumento do $\mathrm{Cd}$ na fração óxidos de $\mathrm{Fe}$ no solo após cultivo. Vários estudos têm mostrado que aplicação de óxidos de Fe e $\mathrm{Mn}$ reduz significativamente a solubilidade de $\mathrm{Cd}$ por aumentar sua retenção nos solos. ${ }^{20}$

Na Tabela 2 encontram-se os teores de Cd extraído nas frações do solo para o solo inicial e após o cultivo. Observa-se que não ocorreram diferenças na fração solúvel + trocável (F1) entre os pontos coletados no solo antes e após a cultura. O mesmo ocorreu para a fração ligada à matéria orgânica (F2), no entanto pode-se notar nesta fração aumento no teor de Cd ligado a matéria orgânica do ponto 7 para o 1 .

$\mathrm{Na}$ fração ligada a óxidos de $\mathrm{Fe}$ e $\mathrm{Al}$ (F3) apenas após o cultivo houve diferença entre os pontos de coleta. Verifica-se que o solo mais contaminado (ponto 1) apresentou maior teor ligado a óxidos após o cultivo do arroz, porém não houve diferença estatística entre o ponto com menor contaminação por $\mathrm{Cd}$ (ponto 7) e o de maior contaminação. Na fração residual (F4), observa-se tendência em diminuir o teor de Cd do ponto 7 para o ponto 1 .

\section{Cobre $(\mathbf{C u})$}

A porcentagem de $\mathrm{Cu}$ nas diversas frações químicas variou no ponto 1 e entre o solo inicial e após o cultivo (Figura 2). A participação da forma solúvel + trocável foi significativamente menor em todos os pontos de coleta. Com relação à fração ligada à matéria orgânica observa-se afinidade do $\mathrm{Cu}$. Autores verificaram reação deste elemento com os grupos $\mathrm{COOH}$ e $\mathrm{OH}$ - fenólicos, simultaneamente, formando complexos altamente estáveis. ${ }^{10}$ Embora a associação do $\mathrm{Cu}$ com a matéria orgânica seja amplamente relatada na literatura, ${ }^{21}$ no presente estudo verificou-se que a sua participação na retenção do $\mathrm{Cu}$ foi pequena $(<10 \%)$, ao contrário do que se encontrou na literatura que a retenção do $\mathrm{Cu}$ pela matéria orgânica foi cerca de $40 \%{ }^{22}$

$\mathrm{A}$ maior parte do $\mathrm{Cu}$ foi encontrada associada às formas ligadas a óxidos de $\mathrm{Fe}$ e $\mathrm{Al}$ e principalmente à fração residual. Apenas no ponto 1 a porcentagem de $\mathrm{Cu}$ na fração ligada a óxidos de $\mathrm{Fe}$ e $\mathrm{Al}$ foi significativamente maior que a fração residual. A afinidade de $\mathrm{Cu}$ por óxidos de Fe é bem documentada na literatura. ${ }^{23}$

Elementos presente na fração residual são aqueles ligados aos silicatos, óxidos de Fe bem cristalizados e outros minerais mais resistentes, os quais não foram solubilizados nas extrações anteriores. ${ }^{19}$

Tabela 2. Teores de Cd, em mg kg-1, extraído em cada fração de amostra de solo contaminado, antes e após cultivo de arroz

\begin{tabular}{|c|c|c|c|c|c|c|c|c|c|c|c|c|c|c|c|}
\hline \multirow{3}{*}{$\begin{array}{c}\begin{array}{c}\text { Pontos de } \\
\text { coleta de solo }\end{array} \\
1\end{array}$} & \multicolumn{4}{|c|}{ Fração 1} & \multicolumn{3}{|c|}{ Fração 2} & \multicolumn{4}{|c|}{ Fração 3} & \multicolumn{4}{|c|}{ Fração 4} \\
\hline & \multicolumn{2}{|c|}{ I } & \multicolumn{2}{|c|}{ A } & \multicolumn{2}{|c|}{ I } & \multirow{2}{*}{$\frac{\mathrm{A}}{0,3}$} & \multicolumn{2}{|c|}{ I } & \multicolumn{2}{|c|}{ A } & \multicolumn{2}{|c|}{ I } & \multicolumn{2}{|c|}{$\mathrm{A}$} \\
\hline & 0,6 & $a b$ & 0,8 & $a b$ & 0,3 & $\mathrm{a}$ & & 5,9 & $\mathrm{a}$ & 7,7 & $\mathrm{~A}$ & 17,9 & $\mathrm{~d}$ & 8,0 & $\mathrm{~b}$ \\
\hline 2 & 0,7 & $\mathrm{a}$ & 0,8 & $a b$ & 0,2 & $\mathrm{~b}$ & - & 6,5 & $\mathrm{a}$ & 6,2 & $\mathrm{Bc}$ & 15,9 & $\mathrm{f}$ & 7,5 & $\mathrm{bc}$ \\
\hline 3 & 0,6 & $a b$ & 0,7 & $\mathrm{~b}$ & 0,1 & $\mathrm{c}$ & - & 6,0 & $\mathrm{a}$ & 6,2 & $\mathrm{Bc}$ & 13,7 & $\mathrm{~g}$ & 5,3 & $\mathrm{~d}$ \\
\hline 4 & 0,5 & $\mathrm{~b}$ & 0,6 & $\mathrm{~b}$ & 0,1 & $\mathrm{c}$ & - & 5,8 & $\mathrm{a}$ & 6,2 & $\mathrm{Bc}$ & 17,0 & $\mathrm{e}$ & 6,5 & $\mathrm{c}$ \\
\hline 5 & 0,8 & $\mathrm{a}$ & 0,9 & $\mathrm{a}$ & 0,1 & $\mathrm{c}$ & - & 6,0 & $\mathrm{a}$ & 6,6 & $\mathrm{Bc}$ & 19,5 & $\mathrm{c}$ & 10,0 & $\mathrm{a}$ \\
\hline 6 & 0,7 & $a b$ & 0,6 & $\mathrm{~b}$ & - & & - & 5,9 & a & 5,8 & $\mathrm{C}$ & 21,3 & $\mathrm{a}$ & 10,3 & $\mathrm{a}$ \\
\hline 7 & 0,7 & $a b$ & 0,7 & $\mathrm{~b}$ & - & & - & 5,8 & $\mathrm{a}$ & 6,8 & $\mathrm{Ab}$ & 20,2 & $\mathrm{~b}$ & 10,0 & $\mathrm{a}$ \\
\hline
\end{tabular}

Nota: Médias seguidas por letras iguais na coluna não diferem entre si ao nível de 5\% pelo teste de Tukey; I- Solo inicial, antes do cultivo; A - solo após o cultivo de arroz. - Abaixo do limite de detecção de $0,0035 \mathrm{mg} \mathrm{L}^{-1}$ 

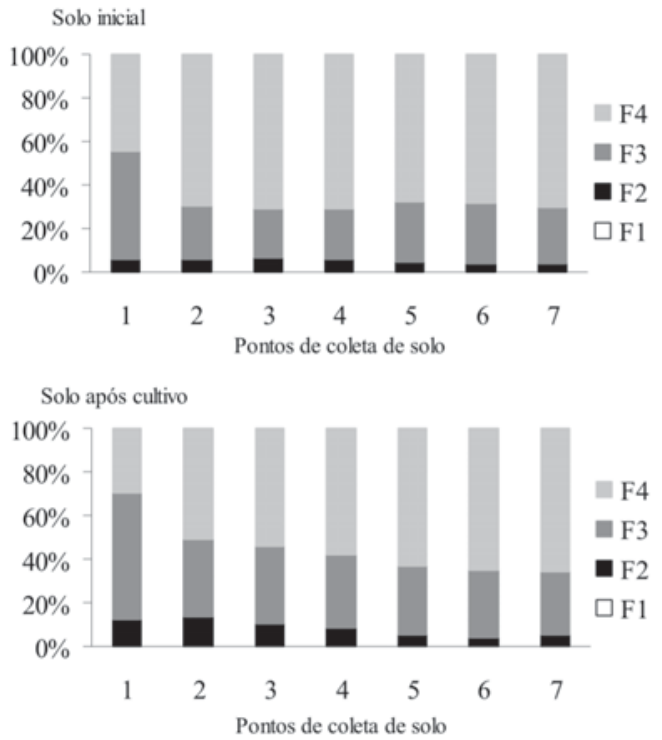

Figura 2. Distribuição do $\mathrm{Cu}$, em porcentagem, nas frações do solo (fração solúvel - F1, fração ligada à matéria orgânica - F2, fração ligada a óxidos - F3 e fração residual - F4) nos pontos de coleta de solo

As maiores concentrações de $\mathrm{Cu}$ em solos encontram-se adsorvidas nos óxidos de Fe e Mn bem cristalizados (hematita, goethita e burnessita) e argilominerais, os quais são os componentes básicos da fração residual. ${ }^{1}$ Alguns autores consideram que a fração residual não é muito significativa, sendo importante apenas para completar o cálculo de balanço de massas dos elementos no solo. ${ }^{24}$ No entanto, esta consideração deve ser importante para solos mais recentemente contaminados, para os quais não houve tempo ou condições termodinâmicas suficiente para a incorporação na rede cristalina dos argilominerais. ${ }^{19}$

Observando o solo inicial e após o cultivo de arroz, nos pontos de coleta, pode-se notar que houve ligeiro aumento na porcentagem do $\mathrm{Cu}$ ligados a óxidos de $\mathrm{Fe}$ e $\mathrm{Al}$, juntamente com a diminuição do residual, sendo mais evidente a partir do ponto 3 , e mais pronunciado no ponto 1 .

Na Tabela 3 encontram-se os teores de Cu extraído nas frações do solo para o solo antes e após o cultivo. Na fração solúvel + trocável obteve-se maior extração no ponto 1 para o solo inicial, enquanto que após o cultivo do arroz, a maior extração foi observada para o ponto 7. Na fração ligada à matéria orgânica nota-se que, tanto para o solo inicial quanto para o solo após o cultivo, houve maior extração no ponto 1 , seguindo tendência em diminuição na extração do ponto 1 para o ponto 7 .

Observando a fração ligada a óxidos de $\mathrm{Fe}$ e $\mathrm{Al}$, verifica-se que a extração de $\mathrm{Cu}$ foi maior no ponto 1 para o solo antes e após o cultivo. $\mathrm{Na}$ fração residual foi obtida maior extração no ponto 1 para o solo inicial, diferindo estatisticamente dos demais pontos, e nos pontos 7 e 6 para o solo após cultivo do arroz.

\section{Manganês (Mn)}

Os processos químicos mais importantes que influenciam o comportamento do Mn no solo e sua disponibilidade para as plantas são os que se relacionam com sua adsorção no solo, aceitando-se, geralmente, que as concentrações na solução do solo e conseqüentemente, a disponibilidade para as plantas são controladas por reações de sorção-dessorção. ${ }^{25} \mathrm{O}$ pH do solo, na maioria dos casos, é o fator mais importante de controle de disponibilidade.

A distribuição do Mn nas diversas formas químicas apresentou variações entre os pontos de coleta no solo após o cultivo de arroz. No solo inicial houve pouca variação das frações entre os pontos (Figura 3). Observa-se menor distribuição de Mn nas frações solúvel + trocável e ligada à matéria orgânica em todos os pontos coletados. Os complexos orgânicos formados com o Mn são de pouca estabilidade, uma vez que o complexo formado com o ácido húmico tem caráter inteiramente eletrostático e os ácidos fúlvicos apresentam limitado número de sítios de complexação específicos para o elemento. ${ }^{26}$

Quantidades consideráveis foram extraídas pelas frações ligadas a óxidos de $\mathrm{Fe}$ e $\mathrm{Al}$ e residual em todos os pontos, demonstrando a importante participação dos óxidos de Fe na adsorção desse elemento. A associação dos metais pesados aos óxidos ocorre preferencialmente por adsorção específica. ${ }^{27}$

Na Tabela 4 encontram-se os teores de Mn extraído nas frações do solo, no solo antes e após o cultivo de arroz. Observa-se na fração solúvel + trocável que o ponto 6 apresentou maior teor de Mn extraído no solo inicial e após o cultivo de arroz.

$\mathrm{Na}$ fração ligada à matéria orgânica as maiores extrações foram observadas para o ponto 6 , no solo inicial, e para o ponto 2 no solo após cultivo do arroz, sendo que este não diferiu estatisticamente dos pontos 3, 4 e 6 . Resultados semelhantes foram observados na fração ligada a óxidos onde, para o solo inicial, o ponto 6 foi o que apresentou maior teor extraído, diferindo dos demais pontos ao nível de probabilidade de $5 \%$. Já para o solo após cultivo, o ponto 1 foi o que apresentou maior teor extraído.

Para a fração residual verifica-se que no solo inicial o ponto 6 apresentou maior teor, diferindo dos demais e para o solo após cultivo, os maiores teores foram para os pontos 5 e 6 .

Tabela 3. Teores de $\mathrm{Cu}$, em mg kg-1 , extraído em cada fração de amostra de solo contaminado, antes e após cultivo de arroz

\begin{tabular}{|c|c|c|c|c|c|c|c|c|c|c|c|c|c|c|c|c|}
\hline \multirow{3}{*}{$\begin{array}{c}\begin{array}{c}\text { Pontos de coleta } \\
\text { de solo }\end{array} \\
1\end{array}$} & \multicolumn{4}{|c|}{ Fração 1} & \multicolumn{4}{|c|}{ Fração 2} & \multicolumn{4}{|c|}{ Fração 3} & \multicolumn{4}{|c|}{ Fração 4} \\
\hline & \multicolumn{2}{|c|}{ I } & \multicolumn{2}{|c|}{ A } & \multicolumn{2}{|c|}{ I } & \multicolumn{2}{|c|}{ A } & \multicolumn{2}{|c|}{ I } & \multicolumn{2}{|c|}{ A } & \multicolumn{2}{|c|}{ I } & \multicolumn{2}{|c|}{ A } \\
\hline & 1,0 & $\mathrm{a}$ & 0,5 & $\mathrm{~d}$ & 15,3 & $\mathrm{a}$ & 28,8 & $\mathrm{a}$ & 134,0 & $\mathrm{a}$ & 138,4 & $\mathrm{a}$ & 121,9 & $\mathrm{a}$ & 71,7 & B \\
\hline 2 & 0,9 & $\mathrm{bc}$ & 0,3 & $\mathrm{f}$ & 7,8 & $\mathrm{~b}$ & 12,4 & $\mathrm{~b}$ & 34,3 & $\mathrm{~d}$ & 32,8 & $\mathrm{e}$ & 97,8 & $\mathrm{~d}$ & 47,3 & $\mathrm{c}$ \\
\hline 3 & 0,8 & $\mathrm{bc}$ & 0,4 & $\mathrm{~d}$ & 6,8 & $\mathrm{bc}$ & 7,3 & $\mathrm{c}$ & 25,8 & $\mathrm{e}$ & 26,1 & $\mathrm{~g}$ & 81,6 & $\mathrm{~g}$ & 39,9 & $\mathrm{c}$ \\
\hline 4 & 0,9 & $a b$ & 0,4 & $\mathrm{e}$ & 6,1 & $\mathrm{~cd}$ & 6,8 & $\mathrm{~cd}$ & 28,2 & $\mathrm{e}$ & 28,4 & $\mathrm{f}$ & 85,5 & $\mathrm{f}$ & 48,8 & $\mathrm{c}$ \\
\hline 5 & 0,8 & $\mathrm{bc}$ & 0,8 & $\mathrm{c}$ & 5,8 & $\mathrm{~cd}$ & 5,9 & de & 39,7 & $\mathrm{c}$ & 38,8 & $\mathrm{~d}$ & 97,8 & $\mathrm{e}$ & 79,2 & $\mathrm{~b}$ \\
\hline 6 & 0,8 & $\mathrm{c}$ & 0,8 & $\mathrm{~b}$ & 5,6 & $\mathrm{~d}$ & 5,0 & $\mathrm{e}$ & 45,7 & $\mathrm{~b}$ & 44,4 & $\mathrm{~b}$ & 114,1 & $\mathrm{~b}$ & 94,4 & $\mathrm{a}$ \\
\hline 7 & 0,8 & $\mathrm{c}$ & 1,0 & $\mathrm{a}$ & 5,1 & $\mathrm{~d}$ & 5,8 & $\mathrm{e}$ & 39,9 & $\mathrm{c}$ & 41,1 & $\mathrm{c}$ & 107,6 & $\mathrm{c}$ & 91,6 & $\mathrm{a}$ \\
\hline
\end{tabular}

Nota: Médias seguidas por letras iguais na coluna não diferem entre si ao nível de 5\% pelo teste de Tukey; I- Solo inicial, antes do cultivo; A - solo após o cultivo de arroz. 

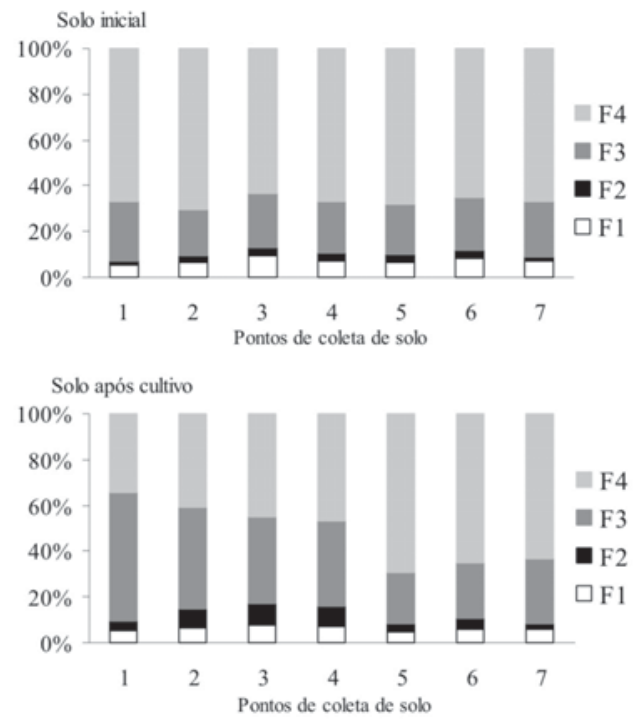

Figura 3. Distribuição do Mn, em porcentagem, nas frações do solo (fração solúvel - F1, fração ligada à matéria orgânica - F2, fração ligada a óxidos - F3 e fração residual - F4) nos pontos de coleta de solo

\section{Chumbo (Pb)}

A distribuição do $\mathrm{Pb}$ nas diversas formas químicas não apresentou variações significativas entre os pontos de coleta de solo (Figura 4). Observa-se predominância de $\mathrm{Pb}$ ligado a óxidos de $\mathrm{Fe}$ e Al, sendo superior, estatisticamente, às demais frações, exceto nos pontos $7 \mathrm{e}$ 6 no solo inicial. Baseados em experimentos, autores relatam que $54 \%$ do $\mathrm{Pb}$ presente nos Latossolos estariam associados à fração óxidos de $\mathrm{Fe},{ }^{19}$ enquanto outros relatam valores em torno de $23 \% .{ }^{10}$ Os dados obtidos neste trabalho não diferem dos citados, variando de 32 a $40 \%$ da soma de todas as frações, dependendo do grau de contaminação, chegando até $51 \%$ no ponto 1 no solo após o cultivo do arroz. Os óxidos de $\mathrm{Fe}$ e $\mathrm{Mn}$ são os principais adsorvedores de $\mathrm{Pb}$ nos solos. ${ }^{28} \mathrm{~A}$ retenção de $\mathrm{Pb}$ aos óxidos de Fe é altamente específica (quimissorção) e o processo resulta na formação de complexos de esfera interna. ${ }^{26}$ Isto faz com que este elemento seja pouco móvel nos solos, uma vez que as reações de quimissorção são menos reversíveis que as de troca iônica.

Observando o solo inicial e após o cultivo pode-se notar que houve ligeiro aumento na porcentagem do $\mathrm{Pb}$ ligados a óxidos de $\mathrm{Fe}$ e $\mathrm{Al}$, semelhante ao que foi observado em outro experimento, no entanto em condições diferentes. ${ }^{29} \mathrm{O}$ autor observou aumento no teor de $\mathrm{Pb}$ na fração óxido à medida que o $\mathrm{pH}$ do solo aumentou o que ocasionaria
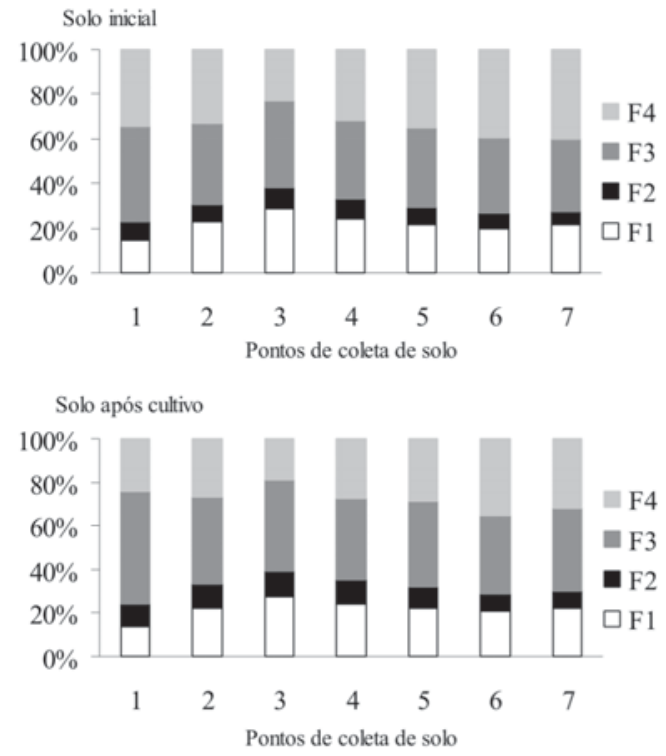

Figura 4. Distribuição do $\mathrm{Pb}$, em porcentagem, nas frações do solo (fração solúvel - F1, fração ligada à matéria orgânica - F2, fração ligada a óxidos - F3 e fração residual - F4) nos pontos de coleta de solo

uma desprotonação, liberando sítios para adsorção de $\mathrm{Pb}$ nos óxidos de Al, explicando assim a maior retenção nesta fração. Autores relatam que estudos com Latossolos brasileiros revelaram que estes possuem grande capacidade de retenção de $\mathrm{Pb}$, sendo que as quantidades adsorvidas dependem do $\mathrm{pH} \cdot{ }^{30} \mathrm{Cabe}$ salientar que considerável fração de $\mathrm{Pb}$ permanece adsorvida mesmo em $\mathrm{pH} 4,5$, mostrando que em valores de $\mathrm{pH}$ mais baixos a disponibilidade de $\mathrm{Pb}$ em Latossolos pode ser reduzida. ${ }^{31}$

Nota-se também que o $\mathrm{Pb}$ na fração trocável manteve-se constante, enquanto que na fração ligada à matéria orgânica ocorreu pequeno aumento após o cultivo. $\mathrm{O} \mathrm{Pb}$ na forma trocável aumenta o risco de contaminação. Existem evidências que a matéria orgânica do solo forma quelatos de baixa solubilidade com o $\mathrm{Pb}^{30}$

$\mathrm{Na}$ Tabela 5 podem-se observar os teores de $\mathrm{Pb}$ extraído das frações do solo, no solo antes e após o cultivo. Verifica-se que na fração solúvel + trocável não houve diferença entre os pontos de coleta no solo inicial. No solo após cultivo pode-se verificar tendência, do maior para o menor teor extraído, do ponto 7 para o ponto 1 .

Já para a fração ligada à matéria orgânica e a fração ligada aos óxidos de $\mathrm{Fe}$ e $\mathrm{Al}$ observa-se tendência contrária, ou seja, aumento no teor extraído do ponto 1 para o ponto 7 . Na fração residual os maiores teores extraídos foram obtidos nos pontos 1 e 6 para o solo após o cultivo.

Tabela 4. Teores de Mn, em mg kg-1 , extraído em cada fração de amostra de solo contaminado, antes e após cultivo de arroz

\begin{tabular}{|c|c|c|c|c|c|c|c|c|c|c|c|c|c|c|c|c|}
\hline \multirow{3}{*}{$\begin{array}{c}\begin{array}{c}\text { Pontos de coleta de } \\
\text { solo }\end{array} \\
1\end{array}$} & \multicolumn{4}{|c|}{ Fração 1} & \multicolumn{4}{|c|}{ Fração 2} & \multicolumn{4}{|c|}{ Fração 3} & \multicolumn{4}{|c|}{ Fração 4} \\
\hline & \multicolumn{2}{|c|}{ I } & \multicolumn{2}{|c|}{ A } & \multicolumn{2}{|c|}{ I } & \multicolumn{2}{|c|}{ A } & \multicolumn{2}{|c|}{ I } & \multicolumn{2}{|c|}{ A } & \multicolumn{2}{|c|}{ I } & \multicolumn{2}{|l|}{ A } \\
\hline & 29,3 & $\mathrm{f}$ & 25,2 & $\mathrm{~cd}$ & 8,3 & $\mathrm{~d}$ & 20,2 & $\mathrm{c}$ & 141,1 & $\mathrm{bc}$ & 268,2 & $\mathrm{a}$ & 361,0 & $\mathrm{f}$ & 165,5 & $\mathrm{c}$ \\
\hline 2 & 38,1 & $\mathrm{e}$ & 23,6 & d & 13,8 & $\mathrm{c}$ & 32,8 & $\mathrm{a}$ & 113,8 & $\mathrm{e}$ & 167,2 & $\mathrm{~b}$ & 397,0 & $\mathrm{c}$ & 153,3 & $\mathrm{c}$ \\
\hline 3 & 49,3 & $\mathrm{~b}$ & 26,5 & $\mathrm{~cd}$ & 20,9 & $\mathrm{~b}$ & 31,4 & $\mathrm{a}$ & 127,8 & $\mathrm{~d}$ & 126,6 & $\mathrm{~d}$ & 337,7 & g & 151,7 & $\mathrm{c}$ \\
\hline 4 & 40,8 & $\mathrm{~cd}$ & 24,9 & $\mathrm{~cd}$ & 19,9 & $\mathrm{~b}$ & 31,5 & $\mathrm{a}$ & 132,4 & $\mathrm{~cd}$ & 132,6 & $\mathrm{~d}$ & 389,7 & d & 164,1 & $\mathrm{c}$ \\
\hline 5 & 42,8 & $\mathrm{c}$ & 35,1 & $\mathrm{~b}$ & 22,6 & $\mathrm{~b}$ & 22,2 & $\mathrm{bc}$ & 145,2 & $\mathrm{~b}$ & 160,1 & $\mathrm{bc}$ & 453,8 & $\mathrm{~b}$ & 486,5 & $\mathrm{a}$ \\
\hline 6 & 58,9 & $\mathrm{a}$ & 44,1 & $\mathrm{a}$ & 28,2 & $\mathrm{a}$ & 29,9 & $a b$ & 163,9 & $\mathrm{a}$ & 172,5 & $\mathrm{~b}$ & 468,2 & $\mathrm{a}$ & 465,2 & $\mathrm{a}$ \\
\hline 7 & 39,4 & de & 29,2 & $\mathrm{c}$ & 12,3 & $\mathrm{~cd}$ & 14,2 & $\mathrm{c}$ & 136,4 & bcd & 143,4 & $\mathrm{~cd}$ & 382,3 & $\mathrm{e}$ & 321,8 & $\mathrm{~b}$ \\
\hline
\end{tabular}

Nota: Médias seguidas por letras iguais na coluna não diferem entre si ao nível de 5\% pelo teste de Tukey; I- Solo inicial, antes do cultivo; A - solo após o cultivo de arroz. 


\section{Zinco (Zn)}

A porcentagem de $\mathrm{Zn}$ nas diversas frações químicas apresentou variação, sendo esta mais evidente no ponto 1 , tanto no solo inicial quanto após o cultivo (Figura 5). Em todos os pontos o Zn esteve mais associado às formas ligadas a óxidos de Fe e residual. Em estudos sobre os efeitos das propriedades do solo na adsorção de $\mathrm{Zn}$, verificou-se que os solos com elevados teores de argila silicatada possuem maior capacidade de adsorção de $\mathrm{Zn}$ que solos arenosos. ${ }^{9}$ Outros pesquisadores relatam que 14 a $38 \%$ do Zn presente no solo está associado com óxidos de Fe e Al. ${ }^{1,19}$ No presente trabalho a variação ficou entre 12 e $24 \%$ no solo inicial, 15 e $52 \%$ no solo após cultivo do arroz. Metais pesados de origem antropogênica são potencialmente mais móveis que aqueles inerentes ao material geológico componente da matriz do solo. ${ }^{1}$

Muitos estudos referentes à adsorção e retenção de $\mathrm{Zn}$ revelam que minerais de argila e a matéria orgânica presente no solo são capazes de reter esse elemento muito fortemente, ${ }^{32}$ enquanto outros relatam pequena participação do $\mathrm{Zn}$ na fração trocável. ${ }^{33} \mathrm{~A}$ matéria orgânica é um constituinte do solo que apresenta íntima relação com metais pesados. Estima-se que aproximadamente 1,5 a 2,3\% do Zn presente nos solos estejam associados à matéria orgânica. ${ }^{1}$ No presente estudo foi obtida uma grande variação na participação da matéria orgânica na retenção do $\mathrm{Zn}$, de 0,8 a $14 \%$ no solo inicial e 0 a $18 \%$ no solo após cultivo. Observa-se que o aumento na fração ligada à matéria orgânica se deu do ponto 7 para o ponto 1, tanto no solo inicial quanto após o cultivo, no entanto o teor de matéria orgânica no solo em todos os pontos coletados manteve-se praticamente constante (Tabela 1). Uma explicação para tal resultado poderia ser pelo fato de que os mecanismos de adsorção e retenção de metais pesados, em solos contaminados com vários elementos, são extremamente competitivos e os elementos com maior afinidade pela matéria orgânica podem ter deslocado o $\mathrm{Zn}$ dos sítios de adsorção. Os teores de $\mathrm{Zn}$ no solo são menores no ponto 7 aumentando gradualmente até o ponto 1 , tanto no solo inicial (Tabela 1) quanto no solo após os cultivos (Tabela 6), enquanto que elementos, como o $\mathrm{Cu}$, que têm alta afinidade com a matéria orgânica, mantêm-se altos em todos os pontos coletados.

A participação da forma solúvel + trocável foi significativamente menor em todos os pontos de coleta de solo, no entanto observa-se aumento na sua participação do ponto 7 para o ponto 1 .

Observando o solo inicial e após o cultivo nos pontos coletados pode-se notar que houve ligeiro aumento na porcentagem do $\mathrm{Zn}$ ligados a óxidos de $\mathrm{Fe}$ e $\mathrm{Al}$, sendo mais evidente nos pontos 1 e 4 . O mesmo foi observado para o Zn trocável + solúvel e ligado à matéria orgânica, conseqüentemente houve diminuição do $\mathrm{Zn}$ residual. A relação entre $\mathrm{pH}$ e disponibilidade do $\mathrm{Zn}$ é bem enfatizada por outros pesquisadores. ${ }^{26,34} \mathrm{~A}$ diferença de $\mathrm{pH}$ entre o solo inicial e após o cultivo, que pode ser justificada pela reação das raízes com o solo,
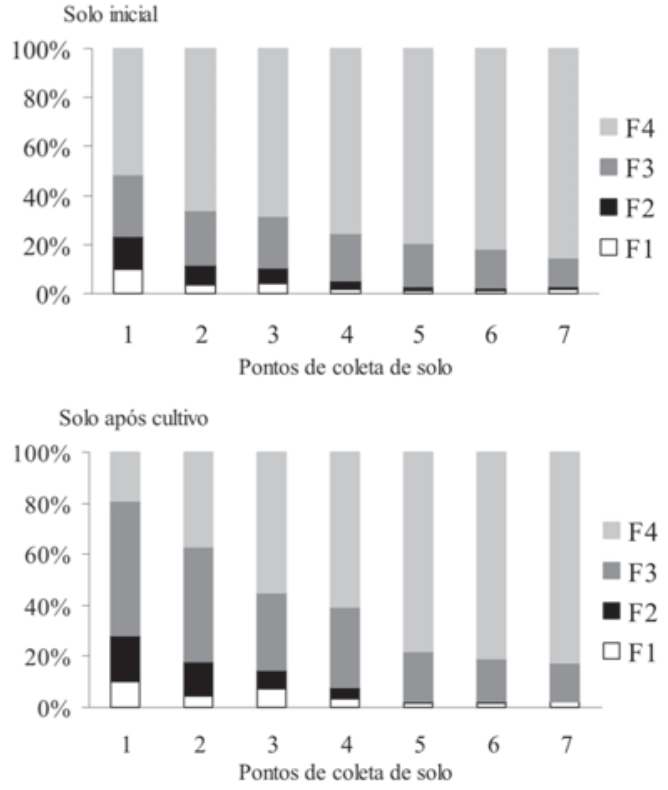

Figura 5. Distribuição do Zn, em porcentagem, nas frações do solo (fração solúvel - F1, fração ligada à matéria orgânica - F2, fração ligada a óxidos - F3 e fração residual - F4) nos pontos de coleta de solo

influenciou os teores de $\mathrm{Zn}$ disponível. O decréscimo de uma unidade de $\mathrm{pH}$ aumenta a solubilidade dos metais pesados em dez vezes. ${ }^{35}$

Na Tabela 6 observam-se os teores de Zn extraídos das frações do solo, no solo antes e após o cultivo. Verifica-se que para as frações solúvel + trocável, ligada à matéria orgânica e ligada a óxidos de Fe e $\mathrm{Al}$ apresentou maior extração no ponto 1, local este com maior grau de contaminação por $\mathrm{Zn}$, diminuindo estatisticamente até o ponto 7 , conforme diminui a contaminação (Tabela 1). A mesma tendência não foi observada na fração residual, apesar de a maior extração ter sido verificada também para o ponto 1 .

\section{CONCLUSÕES}

A análise de fracionamento após o cultivo do arroz demonstrou influência das raízes das plantas sobre as frações nas quais os metais pesados estavam ligados, sobretudo nas frações residuais e ligadas a óxidos.

Os maiores teores de $\mathrm{Cd}, \mathrm{Cu}, \mathrm{Mn}$ e $\mathrm{Zn}$ no solo encontram-se nas frações com ligações químicas mais estáveis (ligados a óxidos e residual) sendo as frações trocável + solúvel e orgânica de menor representatividade, em relação ao total encontrado.

Tabela 5. Teores de $\mathrm{Pb}$, em mg kg-1 , extraído em cada fração de amostra de solo contaminado, antes e após cultivo de arroz

\begin{tabular}{|c|c|c|c|c|c|c|c|c|c|c|c|c|c|c|c|c|}
\hline \multirow{3}{*}{$\begin{array}{c}\begin{array}{c}\text { Pontos de coleta de } \\
\text { solo }\end{array} \\
1\end{array}$} & \multicolumn{4}{|c|}{ Fração 1} & \multicolumn{4}{|c|}{ Fração 2} & \multicolumn{4}{|c|}{ Fração 3} & \multicolumn{4}{|c|}{ Fração 4} \\
\hline & \multicolumn{2}{|c|}{ I } & \multicolumn{2}{|c|}{ A } & \multicolumn{2}{|c|}{ I } & \multicolumn{2}{|c|}{ A } & \multicolumn{2}{|c|}{ I } & \multicolumn{2}{|c|}{$\mathrm{A}$} & \multicolumn{2}{|c|}{ I } & \multicolumn{2}{|c|}{$\mathrm{A}$} \\
\hline & 49,0 & $\mathrm{a}$ & 46,2 & $\mathrm{~b}$ & 28,1 & $\mathrm{a}$ & 34,7 & $\mathrm{a}$ & 142,0 & $\mathrm{a}$ & 170,6 & $\mathrm{a}$ & 113,8 & $\mathrm{a}$ & 80,5 & $\mathrm{a}$ \\
\hline 2 & 48,0 & $\mathrm{a}$ & 45,9 & $\mathrm{~b}$ & 16,1 & $\mathrm{~b}$ & 23,1 & $\mathrm{~b}$ & 75,2 & bcd & 82,2 & $\mathrm{~b}$ & 68,7 & e & 55,6 & $\mathrm{~d}$ \\
\hline 3 & 49,5 & $\mathrm{a}$ & 47,4 & $\mathrm{~b}$ & 16,3 & $b$ & 20,9 & $\mathrm{~cd}$ & 67,4 & $\mathrm{~d}$ & 72,3 & $\mathrm{c}$ & 40,3 & $\mathrm{~g}$ & 32,5 & $\mathrm{e}$ \\
\hline 4 & 48,3 & $\mathrm{a}$ & 47,1 & $\mathrm{~b}$ & 16,8 & $\mathrm{~b}$ & 22,4 & $\mathrm{bc}$ & 69,7 & $\mathrm{~d}$ & 72,7 & $\mathrm{c}$ & 63,6 & $\mathrm{f}$ & 54,4 & d \\
\hline 5 & 49,6 & $\mathrm{a}$ & 49,1 & $\mathrm{a}$ & 16,7 & $\mathrm{~b}$ & 21,5 & $\mathrm{bc}$ & 81,3 & $\mathrm{bc}$ & 86,1 & $\mathrm{~b}$ & 78,5 & $\mathrm{~d}$ & 63,0 & $\mathrm{c}$ \\
\hline 6 & 48,9 & $\mathrm{a}$ & 49,6 & $\mathrm{a}$ & 16,0 & $\mathrm{~b}$ & 19,6 & $\mathrm{~d}$ & 82,3 & $\mathrm{~b}$ & 85,6 & $\mathrm{~b}$ & 97,0 & $\mathrm{~b}$ & 83,3 & $\mathrm{a}$ \\
\hline 7 & 49,2 & $\mathrm{a}$ & 50,2 & $\mathrm{a}$ & 13,7 & $\mathrm{c}$ & 17,5 & $\mathrm{e}$ & 74,1 & $\mathrm{~cd}$ & 85,2 & $\mathrm{~b}$ & 91,6 & $\mathrm{c}$ & 72,1 & $\mathrm{~b}$ \\
\hline
\end{tabular}

Nota: Médias seguidas por letras iguais na coluna não diferem entre si ao nível de 5\% pelo teste de Tukey; I- Solo inicial, antes do cultivo; A - solo após o cultivo de arroz. 
Tabela 6. Teores de Zn, em mg kg-1, extraído em cada fração de amostra de solo contaminado, antes e após cultivo de arroz

\begin{tabular}{|c|c|c|c|c|c|c|c|c|c|c|c|c|c|c|c|c|}
\hline \multirow{3}{*}{$\begin{array}{c}\text { Pontos de coleta de } \\
\text { solo }\end{array}$} & \multicolumn{4}{|c|}{ Fração 1} & \multicolumn{4}{|c|}{ Fração 2} & \multicolumn{4}{|c|}{ Fração 3} & \multicolumn{4}{|c|}{ Fração 4} \\
\hline & \multicolumn{2}{|c|}{ I } & \multicolumn{2}{|c|}{$\mathrm{A}$} & \multicolumn{2}{|c|}{ I } & \multicolumn{2}{|c|}{ A } & \multicolumn{2}{|c|}{ I } & \multicolumn{2}{|c|}{ A } & \multicolumn{2}{|c|}{ I } & \multicolumn{2}{|c|}{ A } \\
\hline & 54,3 & $\mathrm{a}$ & 54,3 & $\mathrm{a}$ & 76,8 & $\mathrm{a}$ & 95,2 & $\mathrm{a}$ & 136,1 & $\mathrm{a}$ & 281,7 & $\mathrm{a}$ & 286,4 & $\mathrm{a}$ & 101,8 & $\mathrm{a}$ \\
\hline 2 & 6,2 & $\mathrm{~b}$ & 7,3 & $\mathrm{~b}$ & 16,2 & $\mathrm{~b}$ & 20,0 & b & 41,6 & $\mathrm{~b}$ & 69,0 & $\mathrm{~b}$ & 124,7 & $\mathrm{~b}$ & 57,1 & $\mathrm{c}$ \\
\hline 3 & 4,9 & $\mathrm{bc}$ & 6,1 & $\mathrm{bc}$ & 6,9 & $\mathrm{c}$ & 5,6 & $\mathrm{c}$ & 24,0 & $\mathrm{c}$ & 24,7 & $\mathrm{c}$ & 76,9 & g & 44,5 & $\mathrm{~d}$ \\
\hline 4 & 1,6 & $\mathrm{~cd}$ & 2,5 & $\mathrm{bc}$ & 4,1 & $\mathrm{~d}$ & 3,0 & $\mathrm{~d}$ & 20,2 & $\mathrm{~d}$ & 21,5 & $\mathrm{~cd}$ & 79,8 & $\mathrm{f}$ & 42,1 & $\mathrm{~d}$ \\
\hline 5 & 1,1 & $\mathrm{~d}$ & 1,7 & $\mathrm{c}$ & 2,0 & $\mathrm{e}$ & 0,6 & $\mathrm{e}$ & 18,5 & de & 20,3 & $\mathrm{~cd}$ & 84,0 & $\mathrm{e}$ & 81,9 & $\mathrm{~b}$ \\
\hline 6 & 1,0 & $\mathrm{~d}$ & 1,8 & $\mathrm{c}$ & 1,7 & ef & 0,7 & $\mathrm{e}$ & 17,0 & $\mathrm{e}$ & 17,7 & d & 88,4 & c & 84,5 & $\mathrm{~b}$ \\
\hline 7 & 1,9 & $\mathrm{~cd}$ & 2,5 & $\mathrm{bc}$ & 0,8 & $\mathrm{f}$ & 0,0 & e & 12,3 & $\mathrm{f}$ & 15,3 & $\mathrm{~d}$ & 87,0 & d & 83,9 & $\mathrm{~b}$ \\
\hline
\end{tabular}

Nota: Médias seguidas por letras iguais na coluna não diferem entre si ao nível de 5\% pelo teste de Tukey; I- Solo inicial, antes do cultivo; A - solo após o cultivo de arroz.

$\mathrm{O} \mathrm{Pb}$, apesar de predominar nas frações ligadas a óxidos e residual, oferece grande potencial de contaminação ambiental por também se apresentar nas frações trocável + solúvel e orgânica em teores consideráveis. O mesmo ocorrendo para o $\mathrm{Zn}$, onde os teores totais foram mais elevados.

\section{REFERÊNCIAS}

1. Kabata-Pendias, A.; Pendias, H.; Trace elements in soil and plants, $3^{\text {rd }}$ ed., CRC Press: Boca Raton, 2001.

2. Salt, D. E.; Prince, R. C.; Pickering, I. J.; Raskin, I.; Plant Physiol. 1995, 109, 1427.

3. Grath, S. P.; Chaudri, A. M.; Giller, K. E.; Summary $15^{\text {th }}$ World Congress of Soil Science, Acapulco, México, 1994.

4. Sutherland, R. A.; Tack, F. M. G.; Tolosa, C. A.; Verloo, M. G.; J. Environ. Qual. 2000, 29, 1431.

5. Tessier, A.; Campbell, P. G. C.; Bisson, M.; Anal. Chem. 1979, 51, 844.

6. Costa, A. C. S. da; Almeida, V. de C.; Lenzi, E.; Nozaki, J.; Quim. Nova 2002, 25, 548

7. Vietz Junior, F. G.; J. Agric. Food Chem. 1962, 10, 174

8. Ure, A. M.; Microchym. Acta 1991, 2, 49; Miller, W. P.; Martens, D. C.; Zelazny, L. W.; Soil Sci. Soc. Am. J. 1986, 50, 598.

9. Shuman, L. M.; Soil Sci. 1985, 140, 11.

10. Gomes, P. C.; Fontes, M. P. F.; Costa, L. M.; Mendonça, E. S.; R. Bras. Ci. Solo 1997, 21, 543.

11. Ahnstrom, Z. S.; Parker, D. R.; Soil Sci. Soc. Am. J. 1999, 63, 1650.

12. Embrapa; Sistema Brasileiro de Classificação de Solo, Embrapa Produção de Informação: Brasília, 1999.

13. Raij, B. van; Quaggio, J. A.; Cantarella, H.; Abreu, C. A. Em Os métodos de análise química do sistema IAC de análise de solo no contexto nacional; Raij, B. van; Andrade, J. C.; Cantarella, H.; Quaggio, J. A., eds.; Instituto Agronômico: Campinas, 2001, cap. 8.

14. Nieuwenhuize, J.; Poley-Vos, C. H.; Akker, A. H. van den; Delft, W. Van; The Anal. 1991, 116, 347.

15. Raij, B. van; Cantarella, H.; Quaggio, J. A.; Furlani, A. M. C.; Recomendações de adubação e calagem para o Estado de São Paulo, $2^{a}$ ed., Instituto Agronômico: Fundação IAC: Campinas, 1996.
16. McGrath, S. P.; Cunliffe, C. H.; J. Sci. Food Agric. 1985, 36, 794.

17. Pimentel-Gomes, F.; Curso de Estatística Experimental, Nobel: Piracicaba, 1990.

18. Hsu, P.H. Em Aluminum oxides and oxyhydroxides; Dixon, J. B.; Weed, S. B., eds.; Soil Science Society of America: Madison, 1989, cap. 5.

19. Bertoni, J. C.; Tese de Doutorado, Universidade Federal de Lavras, Brasil, 2003.

20. Mench, M. J.; Didier, V. L.; Loffler, M.; Gomez, A.; Masson, P.; J. Environ. Qual. 1994, 23, 58; Chen, Z. S.; Lee, G. L.; Lui, J. C.; Chemosphere 2000, 41, 235.

21. Alloway, B. J.; Heavy metals in soils, $2^{\text {nd }}$ ed., Blackie Academic \& Professional: London, 1995; McBride, M. B.; Sauvé, S.; Hendershot, W.; Eur. J. Soil Sci. 1997, 48, 337.

22. Illera, V.; Walter, I.; Souza, P.; Cala, V.; Sci. Total Environ. 2000, 255, 29.

23. McKenzie, R. M.; Aust. J. Soil Res. 1980, 18, 61.

24. Wasay, S. A.; Barrington, S.; Tokunaga, S.; J. Soil Contam. 1998, 7, 103.

25. Hogg, D. S.; Mclaren, R. G.; Swift, R. S.; Soil Sci. Soc. Am. J. 1993, 57, 361.

26. Mcbride, M.B.; Environmental chemistry of soils, Oxford University Press: New York, 1994

27. Schwertmann, U.; Taylor, R. M. Em Iron oxides; Dixon, J. B.; Weed, S. B., eds.; Soil Science Society of America: Madison, 1989, cap. 12.

28. Sheppard, M. I.; Thibault, D. H.; Soil Sci. Soc. Am. J. 1992, 56, 415.

29. Santos, G. C. G.; Tese de Doutorado, Universidade de São Paulo, Brasil, 2005.

30. Oliveira, R. de C.; Dissertação de Mestrado, Universidade Federal de Lavras, Brasil, 2002

31. Pierangeli, M. A. P.; Guilherme, L. R. G.; Oliveira, L. R.; Curi, N.; Silva, M. L. N.; Pes. Agropec. Bras. 2001, 36, 1077

32. Lindsay, W. L.; Adv. Agron. 1972, 24, 147.

33. Oliveira, C.; Amaral Sobrinho, N. M. B.; Mazur, N.; R. Bras. Ci. Solo 2003, 27, 171 .

34. Chlopecka, A.; Bacon, J. R.; Wilson, M. J.; Kay, J.; J. Environ. Qual. 1996, 25, 69.

35. Planquart, P.; Bonin, G.; Prone, A.; Massiane, C.; Sci. Total Environ. 1999, 241, 161 